\title{
Urinary Biomarkers Under Investigation for Overactive Bladder Syndrome
}

Authors:

Nayera Guirguis, M.D. *

IUPUI Female Pelvic Medicine and Reconstructive Surgery

1633 N Capitol Ave \# 436,

Indianapolis, IN 46202

$317-962-6600$

$317-962-2409$

E-mail: nrguirguis@yahoo.com

*Corresponding author

Michael Heit, FACOG, M.D., PhD

IUPUI Female Pelvic Medicine and Reconstructive Surgery

1633 N Capitol Ave \# 436,

Indianapolis, IN 46202

$317-962-6600$

317-962-2409

E-mail: mheitmd@gmail.com

\section{Key words:}

Overactive bladder; neurogenic bladder; urinary biomarkers; nerve growth factor; brain-derived nerve growth factor; neurotrophins

This is the author's manuscript of the article published in final edited form as: 


\section{Abstract}

Overactive bladder (OAB) is a symptom syndrome of urinary urgency, frequency, nocturia, and urge incontinence suggestive of lower urinary tract dysfunction. Detrusor overactivity (DO) during urodynamic testing may be the cause of symptoms in $54-70 \%$ of $O A B$ study participants. The identification of urinary biomarkers is warranted due to the high false negative rate of urodynamic testing results for the diagnosis of $\mathrm{DO}$ and for the evaluation of treatment response in study participants with $O A B$ symptoms.

We reviewed the published literature on urinary biomarkers under investigation for $O A B$ with Pub Med up to June 2015 using search keywords that included: "overactive bladder", "nerve growth factor (NGF)", "brain derived nerve growth factor (BDNF)", "prostaglandins", "cytokines" and "CRP".

Current evidence suggests that NGF and BDNF appear to be most promising candidates for urinary biomarkers for the diagnosis and the evaluation treatment response. 


\section{$\underline{\text { Introduction }}$}

Overactive bladder $(O A B)$ is not a disease entity but a conglomerate of complex storage symptoms characterized by "urinary urgency, usually accompanied by frequency and nocturia, with or without urge incontinence in the absence of proven infection or other obvious pathology", suggestive of lower urinary tract dysfunction [1-2]. The pathological sensation of urinary urgency is the key symptom of the overactive bladder syndrome.

The diagnosis of OAB is based on the clinical evaluation of the patient's symptoms. The minimum requirements for the diagnosis include a history, physical examination and urinalysis [3]. This evaluation is often used as a basis for initial treatment. Detrusor overactivity (DO) is the occurrence of involuntary detrusor contractions of variable amplitude and duration during urodynamic testing [2] and may be the cause of symptoms in $54-70 \%$ of OAB study participants [4].

The quest for the identification of relevant urinary biomarkers with high sensitivity is warranted due to the low sensitivity (high false negative rate) of urodynamic testing results for the diagnosis of DO in study participants with OAB symptoms. A deeper knowledge of the mechanisms of urinary urgency may help shed light on the pathophysiology of OAB.

\section{Mechanisms of Urgency}

Physiologic filling signals from the bladder are relayed to the spinal cord by afferent myelinated A $\delta$ fibers and non-myelinated C- fibers running in the hypogastric, pelvic and pudendal nerves. 
The $A \delta$ fiber endings are located in the detrusor smooth muscle layer. They are tension receptors and are considered to be the primary mediator of the physiologic sensation of bladder filling when activated. The C- fiber nerve endings are found in the urothelium and the lamina propria [5]. They are activated by distention as the bladder nears functional capacity, therefore the threshold for $\mathrm{C}$ - fiber activation is greater than $\mathrm{A} \delta$ fiber activation, because of their smaller fiber diameter and non-myelination. The normal micturition reflex is initiated by signals from the A $\delta$ fibers. The C- fibers are not essential for normal voluntary voiding [6].

C fibers can become more mechanosensitive under certain pathologic conditions, leading to urinary urgency and detrusor overactivity. Disease induced changes in C fiber afferent nerve excitability and reflex bladder activity is mediated by neurotrophins [7].

Neurotrophins are growth factors required by the nerve cells for the survival, maintenance and differentiation of sensory and sympathetic neurons. There are four members of the neurotrophin family: nerve growth factor (NGF), brain-derived neurotrophic factor (BDNF), neurotrophin-3 (NT-3), and neurotrophin-4 (NT-4). Neurotrophins act via the family of tyrosine kinase receptors (Trk), with TrkA receptors having an affinity only for NGF and TrkB receptors having an affinity for BDNF [8].

\section{Biomarkers}

Biomarkers are biological indicators that can be objectively measured and evaluated. These indicators can be used to ascertain the presence of a specific disease entity (diagnostic biomarkers), assess its severity or progression (prognostic biomarkers) and evaluate its response to a particular treatment (predictive biomarkers) [9]. The use of urinary biomarkers in 
the diagnosis and the evaluation of treatment response in patients with OAB remains controversial because their clinical value is unclear. Biomarkers have been used to better elucidate the different pathophysiological processes involved in the development of this syndrome and the pharmacological response to therapeutic modalities.

There are several biomarkers that are currently under investigation for OAB including the aforementioned urinary neurotrophins - nerve growth factor (NGF) and brain derived neuropathic factor (BDNF), as well as prostaglandins and cytokines (Table 1).

Table 1. Strength of Evidence

\begin{tabular}{|c|c|c|c|}
\hline Biomarker & Diagnostic & Prognostic & Reference \\
\hline NGF & $* * *$ & $* * *$ & $12,13,14,15,16,17,18,19,20$ \\
\hline BDNF & $* *$ & $* *$ & 13,25 \\
\hline SPGE2 & $* *$ & $* *$ & $32,33,34,35$ \\
\hline CRP & $*$ & $*$ & $36,37,38,40$ \\
\hline CXCL-1/ CXCL-10 & $*$ & $*$ & 39,40 \\
\hline
\end{tabular}

\section{Urinary Nerve Growth Factor}

NGF is a signaling protein that is expressed widely in various cells outside the nervous system. In the urinary tract it is produced by bladder urothelium and smooth muscle [10]. NGF can affect a wide variety of cell types in the bladder but the majority of research has focused on the effects of NGF levels on bladder afferent fibers. Inflammation, spinal cord injury, denervation and mechanical stretch, have been shown to cause a rise in bladder NGF levels [11].

Multiple studies have been performed in humans, in an attempt to better understand the link between urinary NGF levels and lower urinary tract symptoms. Liu et al. correlated urinary NGF levels with age, BMI, menopausal status, and OAB-wet OAB-dry patient symptomatology. The 
urinary nerve growth factor/creatinine (NGF/Cr) levels were noted to be significantly higher in the OAB-wet sub-group $(2.13 \pm 3.87)$ compared to the OAB-dry sub-group $(0.265 \pm 0.59)$ and the control group $(0.07 \pm 0.21)$. Urinary NGF/Cr was not significantly correlated with age $(P=$ $0.088)$ or $\mathrm{BMI}(\mathrm{P}=0.886)$ in women with OAB-dry and OAB-wet [12].

It has been demonstrated that urinary NGF/Cr levels in healthy individuals are consistently low, do not exhibit circadian variation and demonstrate no significant difference between the genders [13]. Urinary NGF increased physiologically in normal subjects at urge to void but remained pathologically elevated in OAB study participants at small bladder volumes without a significant increase at urge sensation. Urine samples were collected th the first sensation of bladder filling (FSF) and at urge sensation (US) in 39 study participants with OAB and 35 controls without lower urinary tract symptoms. Urinary NGF/Cr levels in controls were low at FSF $(0.011 \pm 0.008)$ and were significantly higher at US $(0.086 \pm 0.022, p=0.005)$. Study participants with OAB had significantly higher urinary NGF/Cr levels at both FSF $(0.45 \pm 0.13, p=$ $0.001)$ and US $(1.00 \pm 0.32, p=0.004)$ compared to controls. The difference in urinary NGF/Cr levels between FSF and US in OAB study participants, however, was not significant $(p=0.064)$ [14].

A study by Chuang revealed that urinary NGF/Cr did not reflect changes in bladder conditions based on subjective symptoms but reflected dynamic changes in bladder pathophysiology based on urodynamic findings in study participants who had not been treated for lower urinary tract symptoms in the last 3 months [15]. There was no significant difference in urinary NGF/Cr showed no significant difference among study participants with improved, stable or worse 
bladder conditions based on overactive bladder or urgency symptom severity scale scores. However, urinary NGF/Cr was significantly decreased in study participants with an improved urodynamic diagnosis (mean $0.94 \pm 1.36$ vs. $0.17 \pm 0.19 \mathrm{pg} / \mathrm{mg}, \mathrm{p}=0.02$ ), significantly increased in study participants with a worse urodynamic diagnosis $(0.55 \pm 0.85$ vs. $2.08 \pm 2.81 \mathrm{pg} / \mathrm{mg}$, $p=0.04)$ and showed no change in those with a stable urodynamic diagnosis $(0.98 \pm 2.31$ vs. 0.97 $\pm 1.89 \mathrm{pg} / \mathrm{mg}, \mathrm{p}=0.99$ ).

The use of urinary NGF levels as a potential biomarker for DO was investigated in women with mixed urinary incontinence (stress urinary incontinence with coexistent urge urinary symptoms). Urinary NGF was measured in 38 women with mixed urinary incontinence, 26 women with urodynamic DO only, 21 women with persistent USI after continence surgery, 15 women with de novo DO after continence surgery, and 31 control subjects. The urinary NGF/Cr levels were low in women with pure USI, and significantly higher in women with USI and coexistent DO. The urinary NGF/Cr in the mixed urinary incontinence group was similar to the levels in women with pure DO. The NGF/Cr levels were undetectable in women with persistent USI but were significantly higher in those with de novo DO after continence surgery. A urinary NGF/Cr level of $>0.05$ was found in $9 \%$ of women with USI, $77 \%$ with DO, $81 \%$ with mixed USI and coexisitent DO, and $80 \%$ with de novo DO. This study indicated that NGF could be a potential biomarker for the presence of DO in women with mixed etiologies [16]. Improving $\mathrm{OAB}$ symptoms as measured by the urgency severity scale (USS) correlated with declining urinary NGF/Cr levels in responders to anticholinergic treatment validating NGF/Cr as a potential predictive urinary biomarker. Liu et al. measured urinary NGF levels in 38 controls 
and 70 study participants with OAB symptoms. Study participants with OAB symptoms were treated with tolterodine $4 \mathrm{mg}$ once daily. NGF/Cr levels and USS scores were compared at baseline, 1, 2 and 3 months after tolterodine and 1 month after cessation of treatment. Urinary NGF/Cr levels were significantly reduced at 3 months in 50 responders ( $1.10 \pm 0.26$ before vs. $0.41 \pm 0.09$ after, $p=0.008)$ but not in the 20 non-responders $(1.38 \pm 0.54$ before vs. $1.30 \pm 0.46$ after, $p=0.879$ ). After anti-cholinergic treatment cessation, the urinary NGF/Cr level was elevated in 23 responders $(0.83 \pm 0.33)$ and in five non-responders $(2.72 \pm 1.41)$ [17] Chronic inflammation from systemic illness and medical co-morbidities may play a role in the pathogenesis of OAB through its effect on circulating levels of serum NGF. Lui et al investigated correlations between serum NGF levels in study participants with OAB refractory to anticholinergic therapy, with and without concurrent medical co-morbidities [18]. Serum NGF levels were significantly higher in study participants with $O A B$ and chronic medical comorbidities ( $4.65 \pm 4.21, p=0.014)$ compared with controls $(2.57 \pm 0.88)$, but not in study participants with $\mathrm{OAB}$ without concurrent chronic medical co-morbidities $(2.88 \pm 0.62$, $\mathrm{p}=0.621)$

Urinary NGF levels as a potential predictive urinary biomarker was studied in study participants with DO before and after treatment with intra- detrusor botulinum toxin A (BoNT-A) injections [19]. Urinary NGF levels were measured in 143 study participants with idiopathic DO (IDO) and 100 with neurogenic DO (NDO) who were untreated, well-treated, and failed-treatment with antimuscarinics. Detrusor injections of BoNT-A (100 U for IDO, $200 \mathrm{U}$ for NDO) were given to 24 study participants with IDO and 19 with NDO who had failed antimuscarinic treatment. Thirtyeight participants without lower urinary tract symptoms served as controls. The urinary NGF/Cr 
levels in study participants with DO at baseline and 3 months after BoNT-A injection were compared to controls. . The mean urinary NGF/Cr levels were significantly higher in 66 study participants with untreated IDO $(1.44 \pm 2.66, \mathrm{p}<0.001)$ and in the 59 with untreated NDO $(0.62$ $\pm 1.22, p<0.001)$ compared to controls $(0.005 \pm 0.019)$. Study participants with well-treated IDO or NDO had reduced NGF/Cr levels, compared to those with failed-treatment IDO or NDO did not. Study participants who responded to BoNT-A treatment had significantly reduced urinary NGF/Cr levels in both the IDO $(0.07 \pm 0.12, p=0.025)$ and NDO $(0.096 \pm 0.17, p=0.033)$ groups compared to baseline levels. However, the NGF levels remained significantly higher at 3 months in 7 IDO $(1.01 \pm 1.25)$ and 5 NDO $(1.64 \pm 2.39)$ study participants who failed BoNT-A treatment.

Shalom studied the use of urinary NGF as a potential predictive biomarker for assessment of the therapeutic response to peripheral nerve evaluation (PNE). Urinary NGF and creatinine levels in 23 study participants with symptomatic DO who failed anticholinergic treatment were compared to controls at baseline and 5 days after a PNE trial. Study participants with DO had significantly higher baseline urinary NGF levels (corrected for creatinine) compared with controls $(19.82 \mathrm{pg} / \mathrm{mg}$ vs $7.88 \mathrm{pg} / \mathrm{mg}, \mathrm{P}<.002)$. Urinary NGF levels significantly decreased from $17.23 \mathrm{pg} / \mathrm{mg}$ to $9.24 \mathrm{pg} / \mathrm{mg}(\mathrm{P}<.02)$ after PNE in symptomatic DO study participants [20].

Urinary NGF levels are higher in study participants with $O A B$ and are responsive to change after effective therapy making it an attractive candidate for both a diagnostic and predictive urinary biomarker. However, NGF has also been shown to be elevated in study participants with acute bacterial cystitis, urinary tract stones or urothelial cell carcinoma that were not associated with 
the presence of OAB symptoms suggesting a low test specificity [21]. As a result, care must be taken to exclude these other etiologies prior to a diagnosis of OAB being made.

\section{Brain-Derived Nerve Growth Factor}

Brain-derived neurotrophic factor (BDNF) is a tissue-derived trophic protein that is found abundantly in the central nervous system. Its synthesis occurs in small-to-medium dorsal root ganglia and stored in dense-core synaptic vesicles. There is growing evidence to suggest that BDNF plays an important role in pain and inflammatory processes. Noxious stimuli have been shown to induce the release of BDNF in the dorsal horn of the spinal cord [22] and intra-thecal injection of BDNF has been shown to decrease the threshold to noxious stimuli [23]. In addition, it has been shown that intrathecal injections of NGF increased BDNF levels in a sub-population of cells that expressed the TrkA receptor [24]. Studies investigating the role of BDNF in bladder inflammation and potentially $O A B$ have been conducted given its involvement in pain and inflammatory processes.

Wang et al [25] compared the diagnostic performance of urinary BDNF to NGF in 90 study participants diagnosed with OAB based on the International Continence Society criteria. Results revealed that BDNF/Cr and NGF/Cr levels were increased in the $90 \mathrm{OAB}$ women compared with controls (BDNF/Cr: 11.95 vs. $1.65, p<0.001 ; N G F / C r: 0.15$ vs. $0.08, p<0.001$ ). There was an 80-fold magnitude difference between BDNF/Cr and NGF/Cr levels in study participants with $O A B$ and yet there was still a significant positive correlation between both $\mathrm{BDNF} / \mathrm{Cr}$ and NGF/Cr levels and symptom severity, as measured by an overactive bladder symptom scale. The sensitivity (88.9\%) and specificity (100.0\%) of tests measuring urinary 
$\mathrm{BDNF} / \mathrm{Cr}$ levels (cut off at $\geq 4.6$ ) were significantly higher than tests measuring $\mathrm{NGF} / \mathrm{Cr}$ levels(cut off at $\geq 0.14$, sensitivity: $54.4 \%$ and specificity: $95.6 \%$ ) for the diagnosis of $O A B$ in symptomatic women.

Antunes- Lopes et al [13] compared urinary NGF and BDNF levels in treatment naïve study participants with $O A B$ after non-pharmacologic and pharmacologic interventions to determine whether lifestyle modification had an influence on urinary neurotrophic factor levels. After lifestyle modifications there was a non-significant decrease in NGF/Cr (mean $488.5 \pm 591.8$ to $319.7 \pm 332.3, p=0.318)$ but a significant decrease in $\mathrm{BDNF} / \mathrm{Cr}$ (mean $628.1 \pm 590.5$ to $432.5 \pm$ 589.0, $p=0.033$ ) compared to pre-treatment levels. After anticholinergic treatment the $\mathrm{NGF} / \mathrm{Cr}$ and BDNF/Cr levels further decreased to a mean of $179.8 \pm 237.9$ and $146.6 \pm 264.9$ (vs baseline $p=0.008$ and $<0.001$, respectively).

The use of BDNF as a potential urinary biomarker is promising. BDNF levels are higher in study participants with $\mathrm{OAB}$ and tests measuring $\mathrm{BDNF} / \mathrm{Cr}$ levels are responsive to change after treatment. The superior diagnostic performance of BDNF relative to NGF may substantiate its use as a more effective diagnostic and predictive urinary biomarker. However, caution must be used when interpreting these results as BDNF has been investigated as a marker in PBS/ ICS (26, 27 , and 28) suggesting a role as a chronic inflammation predictor.

\section{Prostaglandins}

Prostaglandins (PG) are autacoids that are derived from fatty acids (arachidonic acid) that act like local hormones with a brief duration near the site of synthesis. They are responsible for and implicated in a wide range of physiological events and pathological mechanisms. There are 
four principle types of PG's: PGE2 (responsible for arterial dilation and vascular permeability), PGF2A (vasoconstriction), PGD2 (produced by mast cells and in the CNS and plays a role in pain perception), and prostacyclin (potent vasodilator and inhibitor of platelet aggregation). PGs are locally synthesized in the detrusor muscle and mucosa. This synthesis is influenced and initiated by smooth muscle stretch, nerve stimulation, mucosal damage and inflammatory mediators. PGs are implicated in the premature activation of the normal micturition reflex by decreasing the threshold of the stimuli necessary to trigger a bladder contraction through capsaicin sensitive afferent nerve stimulation [29, 30, and 31]

PG may be increased in study participants with $O A B$ and as a result may have the potential to be used a diagnostic urinary biomarker. Kim et al [32] investigated the effect of pathological changes in the bladder (OAB) on urinary levels of PG's and NGF in sixty-five women with OAB compared to twenty without bladder symptoms. Urinary PGE2 and PGF2 $\alpha$ were significantly increased in study participants with $O A B$ compared with controls $(1.54 \pm 0.15$ and $0.94 \pm 0.17$ versus $0.45 \pm 0.15$ and $0.28 \pm 0.10$, respectively, $p<0.05$ ). There was no statistical difference in urinary PGI2 levels in OAB study participants compared to controls. Urinary PGE2 levels were positively correlated with volume at first desire to void and maximum cystometric capacity $(r=0.445, p<0.05)$ in study participants with $O A B$ suggesting that generation of PG's can be induced by bladder stretching and distention.

Liu et al [33] investigated whether the measurement of PGE2 levels could differentiate between study participants with $O A B$ and ICS/PBS syndromes. Urinary PGE2/Cr levels did not differ between study participants with DO based on urodynamic results, over active bladder 
symptoms without urodynamic evidence, and interstitial cystitis/bladder pain syndrome(IC/BPS) compared to controls.

Cho et al. [34] investigated the effect of anticholinergic treatment on changes in urinary NGF and PGE2 levels in thirty study participants with OAB compared to fifteen healthy women. Urinary NGF and PGE2 levels were significantly higher in OAB study participants than in the controls. However, tests measuring PGE2 levels were not responsive to change after treatment with anticholinergics.

Hegele et al [35] analyzed PGE2 blood levels (sPGE2) in study participants with OAB before and 4 weeks after BoNT-A therapy. Significantly higher sPGE2 levels were detected in study participants with OAB compared to the control group (2750 pg/ml vs. $1674 \mathrm{pg} / \mathrm{ml}, \mathrm{p}<0.005)$. In study participants treated with BoNT-A, sPGE2 levels decreased after treatment $(2995 \mathrm{pg} / \mathrm{ml}$ versus $486 \mathrm{pg} / \mathrm{ml}, \mathrm{p}<0.005)$. Decrease in sPGE2 levels were positively correlated with the mean duration of drug effect (9 month). Study participants with a shorter drug effect ( $<9$ months) had less of a SPGE2 decrease compared to study participants with a drug effect $>9$ months ( $22.2 \%$ vs. $57.4 \%$ decrease, $p<0.05)$. There were 3 non-responders to BoNT-A, 2 of which had an increase in their SPGE2 levels of 51\% compared to baseline.

While urinary PG levels were elevated in study participants with $\mathrm{OAB}$, their use as potential diagnostic and prognostic urinary biomarkers is hindered by the lack of difference in study participants with OAB and ICS/PBS and responsiveness to change after treatment. The use of sPGE2 as a diagnostic and predictive urinary biomarker seems promising. 


\section{$C$ reactive protein and Cytokines}

C- reactive protein (CRP) is a pentameric plasma protein that is produced by hepatocytes. It is an acute phase reactant that increases after the secretion of interlukin- 6 from macrophages and $T$ cells serving as a marker for inflammation. Cytokines are a group of small proteins released by cells for signaling behavior change in neighboring cells in response to infection, and inflammation. CRP and cytokines have been investigated as potential biomarkers for lower urinary tract symptoms because chronic inflammation has been implicated in the development of OAB and ICS/PBS [36].

Chung et al [37] measured serum CRP and urinary nerve growth factor (NGF) levels in 22 study participants with OAB, and 48 study participants with IC/BPS compared to controls. Serum CRP levels were significantly higher in subjects with OAB $(1.83 \pm 2.30 \mathrm{mg} / \mathrm{L}$ vs. $0.59 \pm 0.40 \mathrm{mg} / \mathrm{L}$, $p=0.012$ ) compared to controls but there was no significant difference in CRP level between study participants with OAB and IC/BPS $(p=0.43)$. While CRP levels were elevated in study participants with $O A B$, the magnitude of the increase was not suggestive of systemic infection. Only $13.6 \%$ of OAB and $8.7 \%$ of IC/BPS study participants had a clinically significant elevated serum CRP level (>3 mg/L), suggesting the inflammatory process in OAB or ICS/PBS is localized.

Hsiao et al. [38] determined that median CRP levels were significantly higher in women with overactive bladder $(\mathrm{OAB})$ wet $(0.12 \mathrm{mg} / \mathrm{dl})$ compared to women with bladder hypersensitivity/ 
OAB dry $(0.075 \mathrm{mg} / \mathrm{dl}, \mathrm{p}=0.008)$ and controls $(0.055 \mathrm{mg} / \mathrm{dl}, \mathrm{p}=0.032)$ suggesting a positive correlation between serum CRP and severity of symptoms.

Tyagi et al. [39] investigated the role of select chemokines as a diagnostic biomarker in a subset of study participants with ulcerative and non-ulcerative ICS/PBS. There was a greater than tenfold increase in CXCL-1 levels (mean $170 \pm 60.55$ vs $12.55 \pm 3.38$ pg/ml) and a fivefold increase in CXCL-10 levels (684.7 \pm 68.10 vs $123.3 \pm 45.35$ pg/ml, p <0.001) comparing ulcerative to non-ulcerative ICS/PBS cases. There was a similar twentyfold increase in NGF (60.82 \pm 25.38 vs $3.5 \pm 1.6)$ and a fivefold increase in IL-6 (16.06 \pm 6.99 vs $2.42 \pm 1.94 \mathrm{pg} / \mathrm{ml})$.

These findings were further substantiated in a subset of $O A B$ study participants that were refractory to previous anti-muscarinic treatment. Liu et al. [40] noted that the serum concentrations of CRP, NGF, IL-1 $\beta, I L-6, I L-8$, and TNF- $\alpha$ in OAB-dry and OAB-wet study participants were significantly higher than among the controls.

The use of CRP and cytokines as potential biomarkers for OAB and ICS/PBS remains debatable. While there is a growing body of evidence to suggest that inflammation plays a key role in the development of OAB and ICS/PBS, the measurement of CRP and cytokines as urinary biomarkers has its limitation because it can be easily influenced by any systemic inflammatory process.

\section{Conclusion}

Our review of urinary neurotrophins (NGF and BDNF), prostaglandins, CRP and cytokines as urinary biomarkers of $O A B$ has shed some light on the pathophysiology of $O A B$ and the feasibility of their use as diagnostic and prognostic markers. 
The use of NGF as a reliable diagnostic biomarker is promising since it is sensitive enough to distinguish between controls, $O A B$-dry and $O A B$-wet study participants. NGF levels mirrored urodynamic findings in study participants with $O A B$ revealing significantly higher levels at "first sensation" and "urge sensation" compared to controls but did not reflect changes in bladder conditions based on subjective symptoms (urge severity scale). In addition, NGF levels differentiated study participants with pure USI and and USI with coexistent detrusor overactivity.

The use of urinary NGF as a prognostic biomarker is strengthened by evidence revealing significant reduction after anticholinergic treatment in responders compared to nonrespnders. This was similarly demonstrated after BoNT-A injections and PNE treatments.

Though less studied, urinary BDNF has very similar characteristics to urinary NGF with the advantage of having more sensitive and specific tests of its measurement for the diagnosis of OAB in symptomatic study participants. The superior diagnostic performance of BDNF relative to NGF may validate its use as a more effective diagnostic and predictive urinary biomarker.

Urinary PGE2 and PGF2 $\alpha$ were significantly increased in study participants with OAB compared with controls but could not differentiate between study participants with OAB and ICS/PBS syndromes. Furthermore, PGE2 and PGF2 $\alpha$ levels were not responsive to change after treatment limiting their diagnostic and prognostic value. The use of sPGE2 as a diagnostic and predictive urinary biomarker seems promising because of studies demonstrating significantly decreased levels after BoNT-A injections in study participants with OAB. 
Serum CRP levels were significantly higher in subjects with OAB compared to controls but there was no significant difference between study participants with OAB and IC/BPS thus limiting their diagnostic value. The measurement of CRP and cytokines as urinary biomarkers is limited because it can be easily influenced by any systemic inflammatory process.

In summary, we think that NGF has features that make it a good candidate as a diagnostic and prognostic urinary biomarker. While BDNF is not as extensively studied, its characteristics are promising. PG's, CRP and cytokines have shed light on the pathophysiology of the OAB disease process but their inability to distinguish between OAB syndrome and ICS/PBS will limit their use. 


\section{References:}

1. Abrams, P., L. Cardozo, M. Fall, D. Griffiths, P. Rosier, U. Ulmsten, P. van Kerrebroeck, A. Victor and A. Wein (2002). "The standardisation of terminology of lower urinary tract function: report from the Standardisation Sub-committee of the International Continence Society." Am J Obstet Gynecol 187(1): 116-126.

2. Haylen, B. T., D. de Ridder, R. M. Freeman, S. E. Swift, B. Berghmans, J. Lee, A. Monga, E. Petri, D. E. Rizk, P. K. Sand and G. N. Schaer (2010). "An International Urogynecological Association (IUGA)/International Continence Society (ICS) joint report on the terminology for female pelvic floor dysfunction." Int Urogynecol J 21(1): 5-26.

3. Gormley, E. A., D. J. Lightner, K. L. Burgio, T. C. Chai, J. Q. Clemens, D. J. Culkin, A. K. Das, H. E. Foster, Jr., H. M. Scarpero, C. D. Tessier, S. P. Vasavada, A. American Urological, F. P. M. Society of Urodynamics and R. Urogenital (2012). "Diagnosis and treatment of overactive bladder (non-neurogenic) in adults: AUA/SUFU guideline." J Urol 188(6 Suppl): 2455-2463.

4. Digesu, G. A., V. Khullar, L. Cardozo and S. Salvatore (2003). "Overactive bladder symptoms: do we need urodynamics?" Neurourol Urodyn 22(2): 105-108.

5. De Groat, W.C, Yoshimura, N (2009). "Afferent nerve regulation of bladder function in health and disease". Handb Exp Pharmacol. (194):91-138.

6. Steers, W.D (2002). "Pathophysiology of overactive bladder and urge urinary incontinence". Rev Urol. 4 Suppl 4:S7-S18.

7. Vizzard, M. A. (2000). "Changes in urinary bladder neurotrophic factor mRNA and NGF protein following urinary bladder dysfunction." Exp Neurol 161(1): 273-284

8. Kaplan, D. R., B. L. Hempstead, D. Martin-Zanca, M. V. Chao and L. F. Parada (1991). "The trk proto-oncogene product: a signal transducing receptor for nerve growth factor." Science 252(5005): 554-558. 072.

9. Bhide, A. A., R. Cartwright, V. Khullar and G. A. Digesu (2013). "Biomarkers in overactive bladder." Int Urogynecol J 24(7): 1065-1.

10. Tanner R, Chambers P, Khadra MH, Gillespie JI (2000). "The production of nerve growth factor by human bladder smooth muscle cells in vivo and in vitro." BJU Int 85(9):11151119.

11. Steers, W. D. and J. B. Tuttle (2006). "Mechanisms of Disease: the role of nerve growth factor in the pathophysiology of bladder disorders." Nat Clin Pract Urol 3(2): 101-110. 
12. Liu, H. T., C. Y. Chen and H. C. Kuo (2011). "Urinary nerve growth factor in women with overactive bladder syndrome." BJU Int 107(5): 799-803.

13. Antunes-Lopes, T., R. Pinto, S. C. Barros, F. Botelho, C. M. Silva, C. D. Cruz and F. Cruz (2013). "Urinary neurotrophic factors in healthy individuals and patients with overactive bladder." J Urol 189(1): 359-365.

14. Liu, H. T. and H. C. Kuo (2009). "Urinary nerve growth factor levels are elevated in patients with overactive bladder and do not significantly increase with bladder distention." Neurourol Urodyn 28(1): 78-81.

15. Chuang, F. C., H. T. Liu, L. Y. Wang and H. C. Kuo (2014). "Overactive bladder changes with time: a 5-year longitudinal followup of changes in overactive bladder symptoms, urodynamic studies and urinary nerve growth factor levels." J Urol 192(2): 458-463.

16. Liu, H. T., M. B. Chancellor and H. C. Kuo (2008). "Urinary nerve growth factor level could be a biomarker in the differential diagnosis of mixed urinary incontinence in women." BJU Int 102(10): 1440-1444.

17. **Liu, H. T., M. B. Chancellor and H. C. Kuo (2009). "Decrease of urinary nerve growth factor levels after antimuscarinic therapy in patients with overactive bladder." BJU Int 103(12): 1668-1672.

Urinary NGF level could be a potential biomarker for evaluating therapeutic results of antimuscarinics therapy because changes in the urinary NGF levels were associated with the changes of the Urgency Severity Scale scores after antimuscarinic treatment and discontinued medication.

18. Liu, H. T., Y. H. Jiang and H. C. Kuo (2013). "Increased serum adipokines implicate chronic inflammation in the pathogenesis of overactive bladder syndrome refractory to antimuscarinic therapy." PLoS One 8(10): e76706.

19. ** Liu, H. T., M. B. Chancellor and H. C. Kuo (2009). "Urinary nerve growth factor levels are elevated in patients with detrusor overactivity and decreased in responders to detrusor botulinum toxin-A injection." Eur Urol 56(4): 700-706.

Urinary NGF level is a sensitive biomarker for the diagnosis of IDO and NDO and can be used as a tool to evaluate the therapeutic effect of detrusor BoNT-A injection in patients with detrusor overactivity

20. Shalom, D. F., N. Pillalamarri, X. Xue, N. Kohn, L. R. Lind, H. A. Winkler and C. N. Metz (2014). "Sacral nerve stimulation reduces elevated urinary nerve growth factor levels in women with symptomatic detrusor overactivity." Am J Obstet Gynecol 211(5): 561 e561-565. 
21. Liu, H. T., C. Y. Chen and H. C. Kuo (2010). "Urinary nerve growth factor levels in overactive bladder syndrome and lower urinary tract disorders." J Formos Med Assoc 109(12): 862-878.

22. Lever, I. J., E. J. Bradbury, J. R. Cunningham, D. W. Adelson, M. G. Jones, S. B. McMahon, J. C. Marvizon and M. Malcangio (2001). "Brain-derived neurotrophic factor is released in the dorsal horn by distinctive patterns of afferent fiber stimulation." J Neurosci 21(12): 4469-4477.

23. Groth, R. and L. Aanonsen (2002). "Spinal brain-derived neurotrophic factor (BDNF) produces hyperalgesia in normal mice while antisense directed against either BDNF or trkB, prevent inflammation-induced hyperalgesia." Pain 100(1-2): 171-181.

24. Michael, G. J., S. Averill, A. Nitkunan, M. Rattray, D. L. Bennett, Q. Yan and J. V. Priestley (1997). "Nerve growth factor treatment increases brain-derived neurotrophic factor selectively in TrkA-expressing dorsal root ganglion cells and in their central terminations within the spinal cord." J Neurosci 17(21): 8476-8490.

25. **Wang, L. W., X. M. Han, C. H. Chen, Y. Ma and B. Hai (2014). "Urinary brain-derived neurotrophic factor: a potential biomarker for objective diagnosis of overactive bladder." Int Urol Nephrol 46(2): 341-347.

Urinary BDNF/Cr levels are elevated in women with $\mathrm{OAB}$, significantly associated with symptom severity, and offer better sensitivity than NGF in detecting OAB in subjects without other lower urinary tract disorders.

26. Qiao, L. Y. and M. A. Vizzard (2002). "Cystitis-induced upregulation of tyrosine kinase (TrkA, TrkB) receptor expression and phosphorylation in rat micturition pathways." J Comp Neurol 454(2): 200-211.

27. Pinto, R., B. Frias, S. Allen, D. Dawbarn, S. B. McMahon, F. Cruz and C. D. Cruz (2010). "Sequestration of brain derived nerve factor by intravenous delivery of TrkB-Ig2 reduces bladder overactivity and noxious input in animals with chronic cystitis." Neuroscience 166(3): 907-916.

28. Pinto, R., T. Lopes, B. Frias, A. Silva, J. A. Silva, C. M. Silva, C. Cruz, F. Cruz and P. Dinis (2010). "Trigonal injection of botulinum toxin $A$ in patients with refractory bladder pain syndrome/interstitial cystitis." Eur Urol 58(3): 360-365.

29. Bultitude, M. I., N. H. Hills and K. E. Shuttleworth (1976). "Clinical and experimental studies on the action of prostaglandins and their synthesis inhibitors on detrusor muscle in vitro and in vivo." Br J Urol 48(7): 631-637.

30. Park, J. M., J. B. Schnermann and J. P. Briggs (1999). "Cyclooxygenase-2. A key regulator of bladder prostaglandin formation." Adv Exp Med Biol 462: 171-181. 
31. Maggi, C. A. (1992). "Prostanoids as local modulators of reflex micturition." Pharmacol Res 25(1): 13-20.

32. Kim, J. C., E. Y. Park, S. I. Seo, Y. H. Park and T. K. Hwang (2006). "Nerve growth factor and prostaglandins in the urine of female patients with overactive bladder." J Urol 175(5): 1773-1776; discussion 1776.

33. Liu, H. T., P. Tyagi, M. B. Chancellor and H. C. Kuo (2010). "Urinary nerve growth factor but not prostaglandin E2 increases in patients with interstitial cystitis/bladder pain syndrome and detrusor overactivity." BJU Int 106(11): 1681-1685.

34. Cho, K. J., H. S. Kim, J. S. Koh and J. C. Kim (2013). "Changes in urinary nerve growth factor and prostaglandin E2 in women with overactive bladder after anticholinergics." Int Urogynecol J 24(2): 325-330.

35. Hegele, A., S. Knippschild, C. Frohme, J. Hanze, P. Olbert and R. Hofmann (2014). "Changes in prostaglandin E2 in patients with idiopathic overactive bladder syndrome after botulinum toxin type A treatment: is there a clinical benefit?" BMC Urol 14: 85.

36. Saini, R., R. R. Gonzalez and A. E. Te (2008). "Chronic pelvic pain syndrome and the overactive bladder: the inflammatory link." Curr Urol Rep 9(4): 314-319.

37. Chung, S. D., H. T. Liu, H. Lin and H. C. Kuo (2011). "Elevation of serum c-reactive protein in patients with $O A B$ and IC/BPS implies chronic inflammation in the urinary bladder." Neurourol Urodyn 30(3): 417-420.

38. Hsiao SM, Lin HH, Kuo HC (2012). "The role of serum C-reactive protein in women with lower urinary tract symptoms." Int Urogynecol J. 23(7):935-40.

39. Tyagi, P., K. Killinger, V. Tyagi, J. Nirmal, M. Chancellor and K. M. Peters (2012). "Urinary chemokines as noninvasive predictors of ulcerative interstitial cystitis." J Urol 187(6): 2243-2248.

40. Liu, H. T., H. Lin and H. C. Kuo (2011). "Increased serum nerve growth factor levels in patients with overactive bladder syndrome refractory to antimuscarinic therapy." Neurourol Urodyn 30(8): 1525-1529. 